\title{
Lepton-specific inert two-Higgs-doublet model confronted with the new results for muon and electron $g-2$ anomalies and multilepton searches at the LHC
}

\author{
Xiao-Fang Han, ${ }^{1}$ Tianjun Li, ${ }^{2,3}$ Hong-Xin Wang $\odot,{ }^{1}$ Lei Wang $\odot,{ }^{1, *}$ and Yang Zhang ${ }^{4}$ \\ ${ }^{1}$ Department of Physics, Yantai University, Yantai 264005, People's Republic of China \\ ${ }^{2}$ CAS Key Laboratory of Theoretical Physics, Institute of Theoretical Physics, \\ Chinese Academy of Sciences, Beijing 100190, People's Republic of China \\ ${ }^{3}$ School of Physical Sciences, University of Chinese Academy of Sciences, \\ Beijing 100049, People's Republic of China \\ ${ }^{4}$ School of Physics and Microelectronics, Zhengzhou University, \\ ZhengZhou 450001, People's Republic of China
}

(Received 2 August 2021; accepted 16 November 2021; published 1 December 2021)

\begin{abstract}
Combining the multilepton searches at the LHC, we study the possibilities of accommodating the new data of muon and electron $g-2$ anomalies in the lepton-specific inert two-Higgs-doublet model. We take the heavy $C P$-even Higgs as the $125 \mathrm{GeV}$ Higgs, and find the muon and electron $g-2$ anomalies can be explained simultaneously in the region of $5 \mathrm{GeV}<m_{h}<60 \mathrm{GeV}, 200 \mathrm{GeV}<m_{A}<620 \mathrm{GeV}$, $190 \mathrm{GeV}<m_{H^{ \pm}}<620 \mathrm{GeV}$ for appropriate Yukawa couplings between leptons and inert Higgs. Meanwhile, the model can give a better fit to the data of lepton universality in $\tau$ decays than the SM. Further, the multilepton event searches at the LHC impose a stringent upper bound on $m_{h}, m_{h}<35 \mathrm{GeV}$.
\end{abstract}

DOI: $10.1103 /$ PhysRevD.104.115001

\section{INTRODUCTION}

The Fermilab collaboration presented their new result for muon anomalous magnetic moment which now, combined with the data of the E821 [1,2], amounts to [3]

$$
\Delta a_{\mu}=a_{\mu}^{\exp }-a_{\mu}^{\mathrm{SM}}=(251 \pm 59) \times 10^{-11} .
$$

The experimental value has an approximate $4.2 \sigma$ discrepancy from the SM prediction [4-7]. Besides, the measurement value of the fine-structure constant using ${ }^{133} \mathrm{Cs}$ atoms at Berkeley [8] makes the electron $g-2$ to have $2.4 \sigma$ deviation from the SM prediction $[9,10]$,

$$
\Delta a_{e}=a_{e}^{\exp }-a_{e}^{\mathrm{SM}}=(-87 \pm 36) \times 10^{-14} .
$$

However, the newest measurement result of the fine-structure constant using ${ }^{87} \mathrm{Rb}$ atoms at Laboratoire Kastler Brossel gives a value of $a_{e}$ which is well consistent with the SM prediction [11]. The discrepancy between these two

\footnotetext{
* Corresponding author. leiwang@ytu.edu.cn

Published by the American Physical Society under the terms of the Creative Commons Attribution 4.0 International license. Further distribution of this work must maintain attribution to the author(s) and the published article's title, journal citation, and DOI. Funded by SCOAP ${ }^{3}$.
}

experimental results is not clear. If the Berkeley ${ }^{133} \mathrm{Cs}$ experiment result can be confirmed, it will be challenging to explain muon and electron $g-2$ simultaneously since the two excesses have opposite sign. In this paper, we take the Berkeley ${ }^{133} \mathrm{Cs}$ results seriously and discuss the implication of muon and electron $g-2$ on new physics model.

The lepton-specific two-Higgs-doublet model (2HDM) can explain muon $g-2$ anomaly simply [12-27], but will raise the discrepancy in lepton flavor universality (LFU) in $\tau$ decay [22-24]. In addition, the muon and electron $g-2$ anomalies cannot be explained simultaneously in leptonspecific 2HDM since there is an opposite sign between them. There have been some new physics models which attempt to explain the muon and electron $g-2$ simultaneously [28-55]. In Ref. [32], we propose a lepton-specific inert $2 \mathrm{HDM}$ to explain the muon and electron $g-2$ anomalies, and the key point is that these Yukawa couplings for $\mu$ and $e / \tau$ have opposite sign. In the model, the extra Higgses will decay into leptons mainly. Therefore, the multilepton event searches at the LHC can impose stringent constraints on the model. Motivated by the new results for muon $g-2$ and multilepton event searches at the LHC, we revisit the possibilities of accommodating muon and electron $g-2$ anomalies in the lepton-specific inert 2HDM. In this paper, we study a different scenario from that of Ref. [32], and take the inert $C P$-even Higgs $h$ to be lighter than the SM-like Higgs $H$. In such scenario, a very light $C P$-even Higgs $h$ plays important roles in explaining 
the muon and electron g-2 anomalies, and is expected to more easily avoid the constraints from the multi-lepton searches at the LHC than the scenario of Ref. [32] in which $h$ is heavier than the $125 \mathrm{GeV}$ Higgs.

Our work is organized as follows. In Sec. II we will give a brief introduction on the model. In Sec. III and Sec. IV, we introduce the numerical calculations, and show the allowed and excluded parameter space. Finally, we give our conclusion in Sec. V.

\section{LEPTON-SPECIFIC INERT 2HDM}

We add an inert Higgs doublet field to the SM, and the scalar potential is written as,

$$
\begin{aligned}
\mathrm{V}= & Y_{1}\left(\Phi_{1}^{\dagger} \Phi_{1}\right)+Y_{2}\left(\Phi_{2}^{\dagger} \Phi_{2}\right)+\frac{\lambda_{1}}{2}\left(\Phi_{1}^{\dagger} \Phi_{1}\right)^{2}+\frac{\lambda_{2}}{2}\left(\Phi_{2}^{\dagger} \Phi_{2}\right)^{2} \\
& +\lambda_{3}\left(\Phi_{1}^{\dagger} \Phi_{1}\right)\left(\Phi_{2}^{\dagger} \Phi_{2}\right)+\lambda_{4}\left(\Phi_{1}^{\dagger} \Phi_{2}\right)\left(\Phi_{2}^{\dagger} \Phi_{1}\right) \\
& +\left[\frac{\lambda_{5}}{2}\left(\Phi_{1}^{\dagger} \Phi_{2}\right)^{2}+\text { H.c. }\right] .
\end{aligned}
$$

Here we impose a discrete $Z_{2}$ symmetry under which $\Phi_{2}$ is odd and the SM fields are even. We study the $C P$ conserving case where all $\lambda_{i}$ are real. The two complex scalar doublets with the hypercharge $Y=1$ can be given as

$$
\Phi_{1}=\left(\begin{array}{c}
G^{+} \\
\frac{1}{\sqrt{2}}\left(v+H+i G_{0}\right)
\end{array}\right), \quad \Phi_{2}=\left(\begin{array}{c}
H^{+} \\
\frac{1}{\sqrt{2}}(h+i A)
\end{array}\right) .
$$

The $\Phi_{1}$ field has the vacuum expectation value (VEV) $v=246 \mathrm{GeV}$, and the VEV of $\Phi_{2}$ field is zero. The $Y_{1}$ is determined by the scalar potential minimum condition,

$$
Y_{1}=-\frac{1}{2} \lambda_{1} v^{2} .
$$

The Nambu-Goldstone bosons $G^{0}$ and $G^{+}$are eaten by the gauge bosons. The $H^{+}$and $A$ are the mass eigenstates of the charged Higgs boson and $C P$-odd Higgs boson. Their masses are given as

$m_{H^{ \pm}}^{2}=Y_{2}+\frac{\lambda_{3}}{2} v^{2}, \quad m_{A}^{2}=m_{H^{ \pm}}^{2}+\frac{1}{2}\left(\lambda_{4}-\lambda_{5}\right) v^{2}$.

The $H$ is the SM-like Higgs, and has no mixing with the inert $C P$-even Higgs $h$. Their masses are given as

$m_{H}^{2}=\lambda_{1} v^{2} \equiv(125 \mathrm{GeV})^{2}, \quad m_{h}^{2}=m_{A}^{2}+\lambda_{5} v^{2}$.

The fermions obtain the mass terms from the Yukawa interactions with $\Phi_{1}$,

$-\mathcal{L}=y_{u} \bar{Q}_{L} \tilde{\Phi}_{1} u_{R}+y_{d} \bar{Q}_{L} \Phi_{1} d_{R}+y_{l} \bar{L}_{L} \Phi_{1} e_{R}+$ H.c., where $Q_{L}^{T}=\left(u_{L}, d_{L}\right), L_{L}^{T}=\left(\nu_{L}, l_{L}\right), \tilde{\Phi}_{1}=i \tau_{2} \Phi_{1}^{*}$, and $y_{u}$, $y_{d}$ and $y_{\ell}$ are $3 \times 3$ matrices in family space. In addition, in the lepton sector we introduce the $Z_{2}$ symmetry-breaking Yukawa interactions of $\Phi_{2}$,

$$
\begin{aligned}
-\mathcal{L}= & \frac{\sqrt{2} m_{e}}{v} \kappa_{e} \bar{L}_{1 L} \Phi_{2} e_{R}+\frac{\sqrt{2} m_{\mu}}{v} \kappa_{\mu} \bar{L}_{2 L} \Phi_{2} \mu_{R} \\
& +\frac{\sqrt{2} m_{\tau}}{v} \kappa_{\tau} \bar{L}_{3 L} \Phi_{2} \tau_{R}+\text { H.c. }
\end{aligned}
$$

We can obtain the lepton Yukawa couplings of extra Higgses $\left(h, A\right.$, and $H^{ \pm}$) from Eq. (9). The neutral Higgses $h$ and $A$ have no couplings to $Z Z, W W$. At the tree-level, the SM-like Higgs $H$ has the same couplings to fermions and gauge bosons as the SM.

\section{NUMERICAL CALCULATIONS}

In the lepton-specific 2HDM and aligned 2HDM, $\kappa_{\tau}$ equals to $\kappa_{\mu}$. As a result, the decay $\tau \rightarrow \mu \nu \nu$ will obtain negative contribution from the diagram mediated by the charged Higgs. In the model we take $\kappa_{\mu}$ to be opposite in sign from $\kappa_{\tau}$. Thus, the diagrams mediated by the charged Higgs produce positive contribution to the decay $\tau \rightarrow \mu \nu \nu$, and the model can give better fit to the data of the LUF in the $\tau$ decay. When $\kappa_{\mu}$ is opposite in sign from $\kappa_{\tau}$, the contributions of the $C P$-even ( $C P$-odd) Higgses to muon $g-2$ are positive (negative) at the two-loop level and positive (negative) at one-loop level. Therefore, we take the heavy $C P$-even Higgs as the $125 \mathrm{GeV}$ Higgs, $m_{H}=125 \mathrm{GeV}$, and make the light $C P$-even Higgs $h$ to be light enough to enhance muon $g-2$. Since electron $g-2$ is opposite in sign from muon $g-2$, we will take $\kappa_{e}$ to have a opposite sign from $\kappa_{\mu}$.

In our calculations, we take $\lambda_{2}, \lambda_{3}, m_{H}, m_{h}, m_{A}$ and $m_{H^{ \pm}}$ as the input parameters. According to Eqs. (6), (7), the values of $\lambda_{1}, \lambda_{5}$ and $\lambda_{4}$ can be determined. $\lambda_{2}$ controls the quartic couplings of extra Higgses, and does not affect the observables considered in our paper. We take $\lambda_{3}=$ $\lambda_{4}+\lambda_{5}$ to make the Hhh coupling to be absent.

We scan over several key parameters in the following ranges:

$$
\begin{aligned}
5 \mathrm{GeV} & <m_{h}<60 \mathrm{GeV}, \quad 200 \mathrm{GeV}<m_{A}<800 \mathrm{GeV}, \\
90 \mathrm{GeV} & <m_{H^{ \pm}}<800 \mathrm{GeV}, \\
1 & <\kappa_{\tau}<140, \quad-200<\kappa_{\mu}<-1, \quad 1<\kappa_{e}<500 .
\end{aligned}
$$

In such ranges of $\kappa_{\tau}, \kappa_{\mu}$ and $\kappa_{e}$, the corresponding Yukawa couplings do not become nonperturbative.

The model gives the new contributions to muon $g-2$ via the one-loop diagrams and the two-loop Barr-Zee diagrams involving extra Higgses. For the one-loop contributions [12] we have 


$$
\Delta a_{\mu}^{2 \mathrm{HDM}}(1 \text { loop })=\frac{m_{\mu}^{2}}{8 \pi^{2} v^{2}} \sum_{i} \kappa_{\mu}^{2} r_{\mu}^{i} F_{j}\left(r_{\mu}^{i}\right)
$$

where $i=h, A, H^{ \pm}, r_{\mu}^{i}=m_{\mu}^{2} / M_{j}^{2}$. For $r_{\mu}^{i} \ll 1$ we have

$$
F_{h}(r) \simeq-\ln r-7 / 6, \quad F_{A}(r) \simeq \ln r+11 / 6, \quad F_{H^{ \pm}}(r) \simeq-1 / 6 .
$$

The contributions of the two-loop diagrams with a closed fermion loop are given by

$$
\Delta a_{\mu}^{2 \mathrm{HDM}}(2 \text { loop })=\frac{m_{\mu}^{2}}{8 \pi^{2} v^{2}} \frac{\alpha_{\mathrm{em}}}{\pi} \sum_{i, \ell} Q_{\ell}^{2} \kappa_{\mu} \kappa_{\ell} r_{\ell}^{i} G_{i}\left(r_{\ell}^{i}\right),
$$

where $i=h, A, \ell=\tau$, and $m_{\ell}$ and $Q_{\ell}$ are the mass and electric charge of the lepton $\ell$ in the loop. The functions $G_{i}(r)$ are $[13,14]$

$$
\begin{aligned}
G_{h}(r) & =\int_{0}^{1} d x \frac{2 x(1-x)-1}{x(1-x)-r} \ln \frac{x(1-x)}{r}, \\
G_{A}(r) & =\int_{0}^{1} d x \frac{1}{x(1-x)-r} \ln \frac{x(1-x)}{r} .
\end{aligned}
$$

In our calculation, we also include the contributions of the two-loop diagrams with a closed charged Higgs loop, and find that their contributions are much smaller than those of fermion loop. The calculation of $\Delta a_{e}$ is similar to that of $\Delta a_{\mu}$, and we include the contributions of the two-loop diagrams with closed $\mu$ loop and $\tau$ loop.

The HFAG collaboration reported three ratios from pure leptonic processes, and two ratios from semihadronic processes, $\tau \rightarrow \pi / K \nu$ and $\pi / K \rightarrow \mu \nu$ [56]:

$$
\begin{aligned}
\left(\frac{g_{\tau}}{g_{\mu}}\right) & =1.0011 \pm 0.0015, \quad\left(\frac{g_{\tau}}{g_{e}}\right)=1.0029 \pm 0.0015 \\
\left(\frac{g_{\mu}}{g_{e}}\right) & =1.0018 \pm 0.0014, \quad\left(\frac{g_{\tau}}{g_{\mu}}\right)_{\pi}=0.9963 \pm 0.0027 \\
\left(\frac{g_{\tau}}{g_{\mu}}\right)_{K} & =0.9858 \pm 0.0071
\end{aligned}
$$

with the following definitions

$$
\begin{aligned}
& \left(\frac{g_{\tau}}{g_{\mu}}\right)^{2} \equiv \bar{\Gamma}(\tau \rightarrow e \nu \bar{\nu}) / \bar{\Gamma}(\mu \rightarrow e \nu \bar{\nu}), \\
& \left(\frac{g_{\tau}}{g_{e}}\right)^{2} \equiv \bar{\Gamma}(\tau \rightarrow \mu \nu \bar{\nu}) / \bar{\Gamma}(\mu \rightarrow e \nu \bar{\nu}), \\
& \left(\frac{g_{\mu}}{g_{e}}\right)^{2} \equiv \bar{\Gamma}(\tau \rightarrow \mu \nu \bar{\nu}) / \bar{\Gamma}(\tau \rightarrow e \nu \bar{\nu}) .
\end{aligned}
$$

Where $\bar{\Gamma}$ denotes the partial width normalized to its SM value. The correlation matrix for the above five observables is

$$
\left(\begin{array}{ccccc}
1 & +0.53 & -0.49 & +0.24 & +0.12 \\
+0.53 & 1 & +0.48 & +0.26 & +0.10 \\
-0.49 & +0.48 & 1 & +0.02 & -0.02 \\
+0.24 & +0.26 & +0.02 & 1 & +0.05 \\
+0.12 & +0.10 & -0.02 & +0.05 & 1
\end{array}\right)
$$

In the model, we have the ratios

$$
\begin{aligned}
& \left(\frac{g_{\tau}}{g_{\mu}}\right)^{2} \approx \frac{1+2 \delta_{\text {loop }}^{\tau},}{1+2 \delta_{\text {loop }}^{\mu}}, \\
& \left(\frac{g_{\tau}}{g_{e}}\right)^{2} \approx \frac{1+2 \delta_{\text {tree }}+2 \delta_{\text {loop }}^{\tau}}{1+2 \delta_{\text {loop }}^{\mu}}, \\
& \left(\frac{g_{\mu}}{g_{e}}\right)^{2} \approx 1+2 \delta_{\text {tree }}, \\
& \left(\frac{g_{\tau}}{g_{\mu}}\right)_{\pi}^{2}=\left(\frac{g_{\tau}}{g_{\mu}}\right)_{K}^{2}=\left(\frac{g_{\tau}}{g_{\mu}}\right)^{2} .
\end{aligned}
$$

The $\delta_{\text {tree }}$ and $\delta_{\text {loop }}^{\tau, \mu}$ are respectively corrections from the treelevel diagrams and the one-loop diagrams mediated by the charged Higgs. They are given as $[22,24,25]$

$$
\begin{array}{r}
\delta_{\text {tree }}=\frac{m_{\tau}^{2} m_{\mu}^{2}}{8 m_{H^{ \pm}}^{4}} \kappa_{\tau}^{2} \kappa_{\mu}^{2}-\frac{m_{\mu}^{2}}{m_{H^{ \pm}}^{2}} \kappa_{\tau} \kappa_{\mu} \frac{g\left(m_{\mu}^{2} / m_{\tau}^{2}\right)}{f\left(m_{\mu}^{2} / m_{\tau}^{2}\right)}, \\
\delta_{\text {loop }}^{\tau, \mu}=\frac{1}{16 \pi^{2}} \frac{m_{\tau, \mu}^{2}}{v^{2}} \kappa_{\tau, \mu}^{2}\left[1+\frac{1}{4}\left(H\left(x_{A}\right)+H\left(x_{h}\right)\right)\right],
\end{array}
$$

where $f(x) \equiv 1-8 x+8 x^{3}-x^{4}-12 x^{2} \ln (x), g(x) \equiv 1+$ $9 x-9 x^{2}-x^{3}+6 x(1+x) \ln (x)$ and $H\left(x_{\phi}\right) \equiv \ln \left(x_{\phi}\right)(1+$ $\left.x_{\phi}\right) /\left(1-x_{\phi}\right)$ with $x_{\phi}=m_{\phi}^{2} / m_{H^{ \pm}}^{2}$.

We perform $\chi_{\tau}^{2}$ calculation for the five observables. The covariance matrix constructed from the data of Eq. (16) and Eq. (18) has a vanishing eigenvalue, and the corresponding degree is removed in our calculation. In our discussions we require the value of $\chi_{\tau}^{2}$ to be smaller than the SM value, $\chi_{\tau}^{2}(\mathrm{SM})=12.3$.

The measured values of the ratios of the leptonic $Z$ decay branching fractions are given as [57]:

$$
\begin{aligned}
& \frac{\Gamma_{Z \rightarrow \mu^{+} \mu^{-}}}{\Gamma_{Z \rightarrow e^{+} e^{-}}}=1.0009 \pm 0.0028, \\
& \frac{\Gamma_{Z \rightarrow \tau^{+} \tau^{-}}}{\Gamma_{Z \rightarrow e^{+} e^{-}}}=1.0019 \pm 0.0032,
\end{aligned}
$$

with a correlation of +0.63 . In the model, the width of $Z \rightarrow \tau^{+} \tau^{-}$can have sizable deviation from the SM value due to the loop contributions of the extra Higgs bosons, 
because they strongly interact with charged leptons. The quantities of Eq. (22) are calculated in the model are similar to Refs. [22,24,25].

$\frac{\Gamma_{Z \rightarrow \mu^{+} \mu^{-}}}{\Gamma_{Z \rightarrow e^{+} e^{-}}} \approx 1.0+\frac{2 g_{L}^{e} \operatorname{Re}\left(\delta g_{L}^{2 \mathrm{HDM}}\right)+2 g_{R}^{e} \operatorname{Re}\left(\delta g_{R}^{2 \mathrm{HDM}}\right)}{g_{L}^{e 2}+g_{R}^{e 2}} \frac{m_{\mu}^{2} \kappa_{\mu}^{2}}{m_{\tau}^{2} \kappa_{\tau}^{2}}$,
$\frac{\Gamma_{Z \rightarrow \tau^{+} \tau^{-}}}{\Gamma_{Z \rightarrow e^{+} e^{-}}} \approx 1.0+\frac{2 g_{L}^{e} \operatorname{Re}\left(\delta g_{L}^{2 \mathrm{HDM}}\right)+2 g_{R}^{e} \operatorname{Re}\left(\delta g_{R}^{2 \mathrm{HDM}}\right)}{g_{L}^{e 2}+g_{R}^{e 2}}$.

where the SM value $g_{L}^{e}=-0.27$ and $g_{R}^{e}=0.23 . \delta g_{L}^{2 \mathrm{HDM}}$ and $\delta g_{R}^{2 \mathrm{HDM}}$ are given as

$$
\begin{aligned}
\delta g_{L}^{2 \mathrm{HDM}}= & \frac{1}{16 \pi^{2}} \frac{m_{\tau}^{2}}{v^{2}} \kappa_{\tau}^{2}\left\{-\frac{1}{2} B_{Z}\left(r_{A}\right)-\frac{1}{2} B_{Z}\left(r_{h}\right)-2 C_{Z}\left(r_{A}, r_{h}\right)\right. \\
& \left.+s_{W}^{2}\left[B_{Z}\left(r_{A}\right)+B_{Z}\left(r_{h}\right)+\tilde{C}_{Z}\left(r_{A}\right)+\tilde{C}_{Z}\left(r_{h}\right)\right]\right\}, \\
\delta g_{R}^{2 \mathrm{HDM}}= & \frac{1}{16 \pi^{2}} \frac{m_{\tau}^{2}}{v^{2}} \kappa_{\tau}^{2}\left\{2 C_{Z}\left(r_{A}, r_{h}\right)-2 C_{Z}\left(r_{H^{ \pm}}, r_{H^{ \pm}}\right)\right. \\
& +\tilde{C}_{Z}\left(r_{H^{ \pm}}\right)-\frac{1}{2} \tilde{C}_{Z}\left(r_{A}\right)-\frac{1}{2} \tilde{C}_{Z}\left(r_{h}\right) \\
& +s_{W}^{2}\left[B_{Z}\left(r_{A}\right)+B_{Z}\left(r_{h}\right)+2 B_{Z}\left(r_{H^{ \pm}}\right)\right. \\
& \left.\left.+\tilde{C}_{Z}\left(r_{A}\right)+\tilde{C}_{Z}\left(r_{h}\right)+4 C_{Z}\left(r_{H^{ \pm}}, r_{H^{ \pm}}\right)\right]\right\},
\end{aligned}
$$

where $r_{\phi}=m_{\phi}^{2} / m_{Z}^{2}$ with $\phi=A, h, H^{ \pm}$, and

$$
\begin{gathered}
B_{Z}(r)=-\frac{\Delta_{\epsilon}}{2}-\frac{1}{4}+\frac{1}{2} \log (r) \\
C_{Z}\left(r_{1}, r_{2}\right)=\frac{\Delta_{\epsilon}}{4}-\frac{1}{2} \int_{0}^{1} d x \int_{0}^{x} d y \log \left[r_{2}(1-x)\right. \\
\left.+\left(r_{1}-1\right) y+x y\right], \\
\tilde{C}_{Z}(r)=\frac{\Delta_{\epsilon}}{2}+\frac{1}{2}-r[1+\log (r)]+r^{2}\left[\log (r) \log \left(1+r^{-1}\right)\right. \\
\left.-\operatorname{Li}_{2}\left(-r^{-1}\right)\right]-\frac{i \pi}{2}\left[1-2 r+2 r^{2} \log \left(1+r^{-1}\right)\right]
\end{gathered}
$$

The $125 \mathrm{GeV}$ Higgs $(H)$ has the same tree-level couplings to the fermions and gauge bosons as the SM, and the $H \rightarrow h h$ decay is absent for $\lambda_{3}=\lambda_{4}+\lambda_{5}$. Since the extra Higgses have no coupling to quarks, we can safely neglect the constraints from the meson observable. We employ the 2HDMC [58] to implement theoretical constraints from the vacuum stability, unitarity and couplingconstant perturbativity, as well as the constraints from the oblique parameters $(S, T, U)$. Adopting the recent fit results in Ref. [59], we use the following values of $S, T, U$,
$S=0.02 \pm 0.10, \quad T=0.07 \pm 0.12, \quad U=0.00 \pm 0.09$.

The correlation coefficients are given by

$\rho_{S T}=0.92, \quad \rho_{S U}=-0.66, \quad \rho_{T U}=-0.86$.

HiggsBounds [60] is employed to implement the exclusion constraints from the searches for the neutral and charged Higgs at the LEP at 95\% confidence level.

The extra Higgs bosons are dominantly produced at the LHC via the following electroweak processes:

$$
\begin{gathered}
p p \rightarrow Z^{*} \rightarrow h A, \\
p p \rightarrow W^{ \pm *} \rightarrow H^{ \pm} h, \\
p p \rightarrow W^{ \pm *} \rightarrow H^{ \pm} A, \\
p p \rightarrow Z^{*} / \gamma^{*} \rightarrow H^{+} H^{-}, \\
p p \rightarrow Z \rightarrow \tau^{+} \tau^{-} h .
\end{gathered}
$$

In our scenario, the main decay modes of the Higgs bosons are

$$
\begin{gathered}
h \rightarrow \tau^{+} \tau^{-}, \quad \mu^{+} \mu^{-}, \cdots \cdots, \\
A \rightarrow \tau^{+} \tau^{-}, \quad Z h, \mu^{+} \mu^{-} \cdots \cdots, \\
H^{ \pm} \rightarrow \tau^{ \pm} \nu, \quad W^{ \pm} h, \quad \mu^{ \pm} \nu, \cdots \cdots .
\end{gathered}
$$

We perform simulations for the processes using MG5_aMC-2 . 4 . 3 [61] with PYTHIA6 [62] and DELPHES3.2.0 [63], and impose the constraints from all the analysis at the $13 \mathrm{TeV}$ LHC in the latest CheckMATE2.0.28 [64], as well as analysis we implemented in our previous works $[32,65]$. Besides, we also impose the recently published analyses of searching for events with three or more leptons, with up to two hadronical $\tau$ leptons, using $13 \mathrm{TeV}$ LHC $137 \mathrm{fb}^{-1}$ data [66]. It improves the limits on chargino mass in a simplified SUSY model that wino-like chargino/neutralino decaying to $\tau \mathrm{s}$. The signal regions of $4 \mathrm{lI}, 4 \mathrm{lJ}$, and $4 \mathrm{lK}$ give strongest constraints on our samples, which require 4 leptons with one or two hadronical $\tau$ leptons in the final states, because of the dominated multilepton final states in our model.

\section{RESULTS AND DISCUSSIONS}

After imposing the constraints of "premuon $g-2$ " (denoting the theory, the oblique parameters, the exclusion limits from the searches for Higgs at LEP), in Fig. 1 we show the surviving samples which are consistent with $\Delta a_{\mu}$ and $\Delta a_{e}$ at the $2 \sigma$ level. Both one-loop and two-loop 

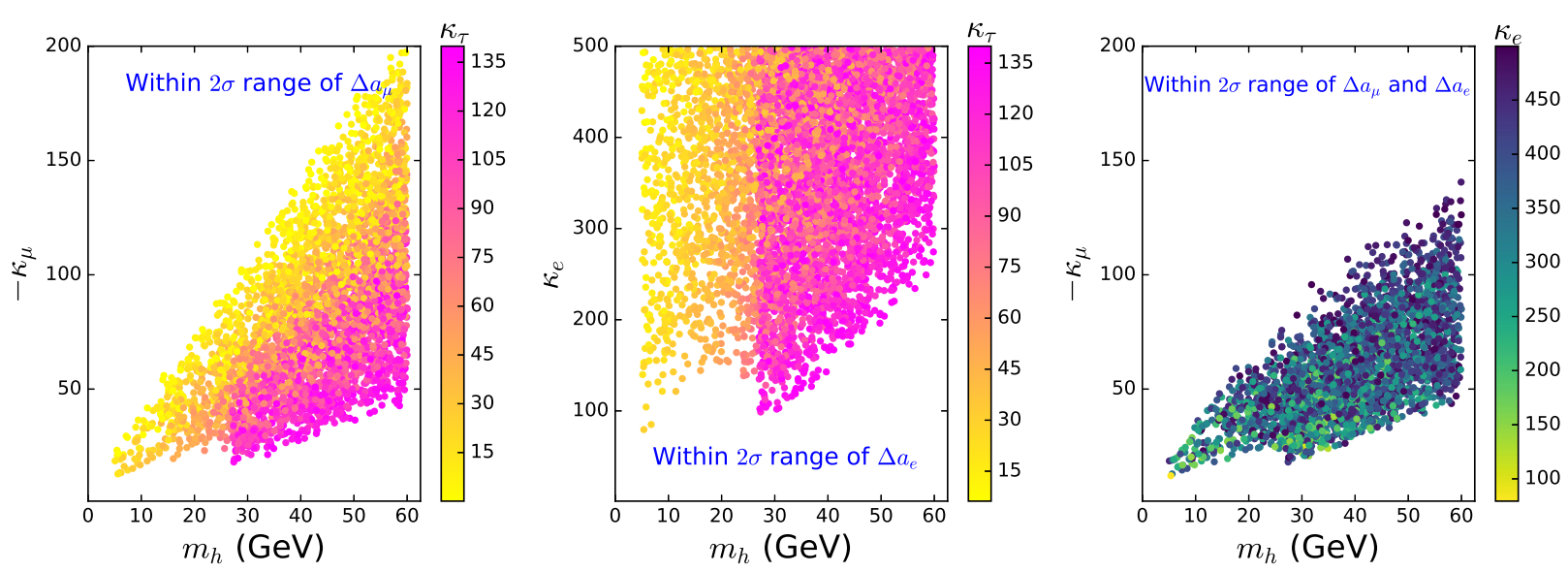

FIG. 1. The samples within $2 \sigma$ ranges of $\Delta a_{\mu}$ (left panel), $\Delta a_{e}$ (middle panel), and both $\Delta a_{\mu}$ and $\Delta a_{e}$ (right panel). All the samples satisfy the constraints of "pre-muon $g-2$ ".

diagrams give positive contributions to $\Delta a_{\mu}$. For $\Delta a_{e}$, the contributions of one-loop are positive and those of twoloop are negative. Only the contributions of two-loop can make $\Delta a_{e}$ to be within $2 \sigma$ range for large enough $\kappa_{\tau} \kappa_{e}$. $\Delta a_{\mu}$ and $\Delta a_{e}$ respectively favor $-\kappa_{\mu}$ and $\kappa_{e}$ to increase with $m_{h}$. For $\kappa_{\tau}=140$ and $m_{h}=60 \mathrm{GeV},-\kappa_{\mu}$ and $\kappa_{e}$ are respectively required to be larger than 40 and 250 . Due to the constraints from the searches for $e e \rightarrow \tau \tau(h) \rightarrow \tau \tau \tau \tau$ at LEP [67], $\kappa_{\tau}$ is required to be smaller than 90 for $m_{h}<27 \mathrm{GeV}$. As a result, the relative large $-\kappa_{\mu}$ and $\kappa_{e}$ are respectively required to explain $\Delta a_{\mu}$ and $\Delta a_{e}$ for $m_{h}<27 \mathrm{GeV}$. The right panel shows that the upper limits of $-\kappa_{\mu}$ within the $2 \sigma$ ranges of both $\Delta a_{\mu}$ and $\Delta a_{e}$ are much smaller than those within the $2 \sigma$ ranges of $\Delta a_{\mu}$.

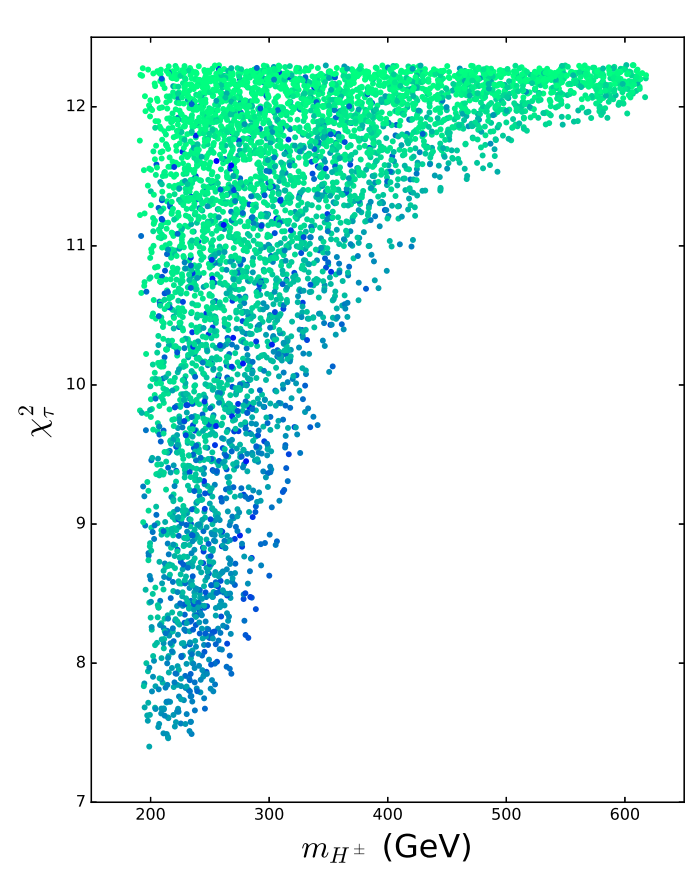

This is because $\kappa_{\tau}$ is required to be large enough to explain $\Delta a_{e}$, and for such large $\kappa_{\tau}, \Delta a_{\mu}$ favors a relative small $\kappa_{\mu}$.

After imposing the constraints of pre-muon $g-2$, we show the surviving samples with $\chi_{\tau}^{2}<12.3$ in Fig. 2 . Because $\kappa_{\mu}$ is opposite in sign from $\kappa_{\tau}$, the second term of $\delta_{\text {tree }}$ in Eq. (20) is positive, which gives a well fit to $g_{\tau} / g_{e}$. Such case is not realized in the lepton-specific 2HDM and aligned 2HDM. From Fig. 2, we find that $\chi_{\tau}^{2}$ increases with $m_{H^{ \pm}}$, and obtains a relative small value for the moderate $-\kappa_{\mu} \kappa_{\tau}$. The $\chi_{\tau}^{2}$ can be as low as 7.4, which is much smaller than the SM value.

In Fig. 3 we show the surviving samples after imposing the constraints of premuon $g-2, \Delta a_{\mu}, \Delta a_{e}, \chi_{\tau}^{2}<12.3$,

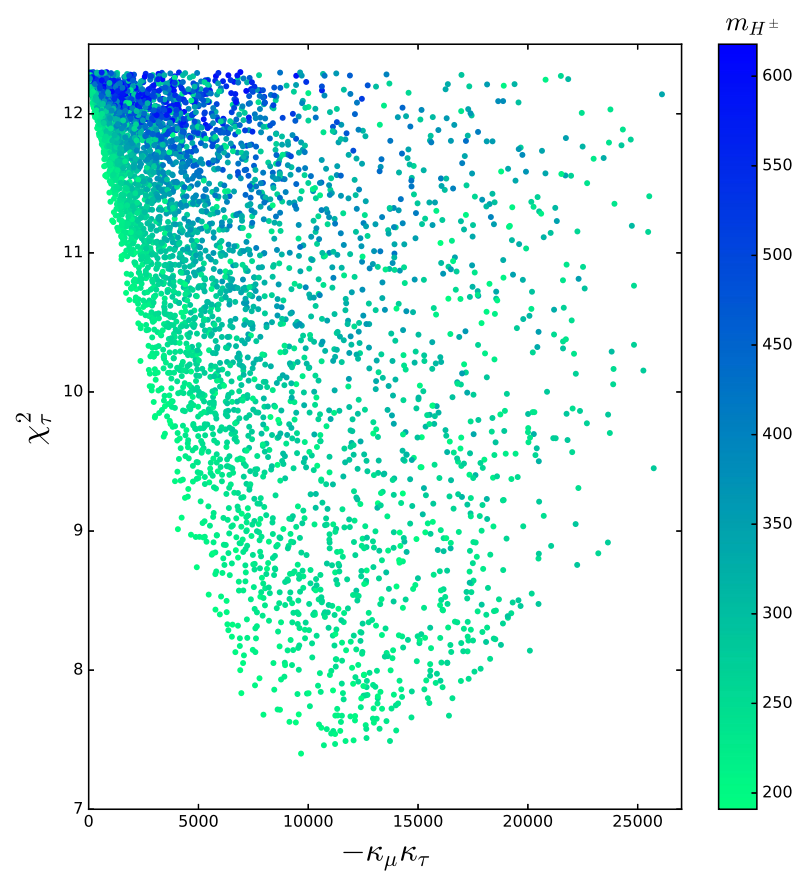

FIG. 2. The surviving samples fit the data of LFU in $\tau$ decay with $\chi_{\tau}^{2}<12.3$. All the samples satisfy the constraints of "premuon $g-2$ ". 

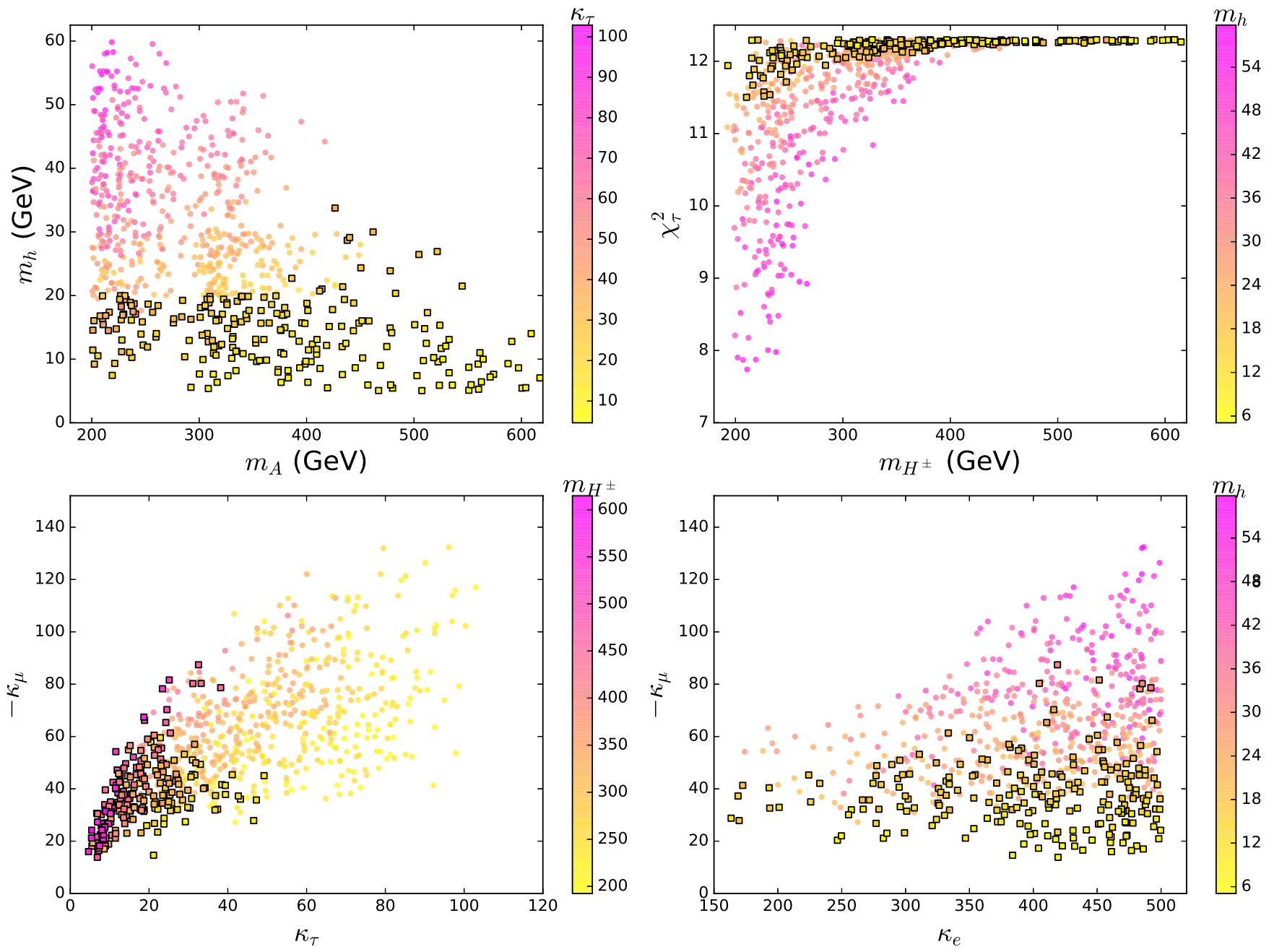

FIG. 3. The allowed samples (squares) and excluded samples (bullets) by the direct search limits from the LHC at $95 \%$ confidence level. All the samples satisfy the constraints of pre-muon $g-2, \Delta a_{\mu}, \Delta a_{e}, \chi_{\tau}^{2}<12.3$, and $Z$ decays.

$Z$ decays, and the direct searches at LHC. The Fig. 3 shows that the points with relatively larger $m_{A} / m_{H^{ \pm}}$or lower $m_{h}$ can escape the direct searches. The production cross sections at the LHC decrease with heavier $A / H^{ \pm}$, and this region can be further detected with higher luminosity and collision energy. For the light $h$, the $\tau$ s from $h$ in decays become too soft to be distinguished at detector, while the $\tau$ s from $h$ in $A / H^{ \pm}$decays are collinear because of the large mass splitting between $h$ and $A / H^{ \pm}$. Meanwhile, the $A / H^{ \pm} \rightarrow$ $h Z / W^{ \pm}$decay modes dominate the $A / H^{ \pm}$decays in the low $m_{h}$ region. Thus, in the region of $m_{h}<20 \mathrm{GeV}$, the acceptance of above signal region for final state of two collinear $\tau+Z / W$ boson quickly decreases.

The upper-right panel of Fig. 3 shows that the $\chi_{\tau}^{2}$ is required to be closed to the SM value since the multilepton event searches at the LHC favor large $m_{H^{ \pm}}$or small $m_{h}$. For small $m_{h}$, the muon and electron anomalies favor small $\kappa_{\tau}$ and $-\kappa_{\mu}$. As a result, the new contribution to $\chi_{\tau}^{2}$ are sizably reduced. The $\chi_{\tau}^{2}$ is allowed to be as low as 11.5 for $m_{H^{ \pm}}$around $200 \mathrm{GeV}$, which is visibly smaller than the SM value, 12.3. The Fig. 3 shows that the parameters of the Yukawa couplings are favored in the region of $15<-\kappa_{\mu}<90,5<\kappa_{\tau}<50$, and $160<\kappa_{e}<500$.

We introduce explicit $Z_{2}$ breaking terms in the Eq. (9). For a natural theory model, such breaking terms should be tiny if the theory respects the original symmetry. Although the $\kappa_{e}, \kappa_{\mu}$, and $\kappa_{\tau}$ are substantial, the breaking terms of Eq. (9) still can be significantly suppressed by $\frac{m_{e}}{v}, \frac{m_{\mu}}{v}$, and $\frac{m_{\tau}}{v}$. In addition, Fig. 3 shows that $\kappa_{e}, \kappa_{\mu}$, and $\kappa_{\tau}$ are allowed to have very small values for a very light $h$. Especially for that $\kappa_{\tau}$ is allowed to be smaller than 10. Therefore, for a light $h$, we may introduce the tiny breaking terms to accommodate the LFU in $\tau$ decay, the muon and electron $g-2$ anomalies.

In the Eq. (9), we make the lepton-flavor-conserving (violation) terms to break (respect) the $Z_{2}$ symmetry. Such case may be naturally achieved by introducing an additional $Z_{4}^{\prime}$ symmetry. Under $Z_{4}^{\prime}$ symmetry, the three generation lepton fields respectively have charge $i,-i,-1$, and other fields including $\Phi_{2}$ have charge 1 . Thus, in the model with explicit $Z_{2}$ breaking, the lepton-flavor-conserving 
(violation) terms are still allowed (forbidden) by the additional $Z_{4}^{\prime}$ symmetry.

In order to avoid the constraint from the $H \rightarrow h h$ decay, we simply take $\lambda_{3}=\lambda_{4}+\lambda_{5}$ to make the tree-level Hhh coupling to be absent. The Hhh coupling can be corrected at one-loop by the exchange of SM leptons in the loop, and the correction is proportional to $\frac{1}{16 \pi^{2}}\left(\frac{m_{\ell}}{v}\right)^{3} \kappa_{\ell}^{2}$. When $\kappa_{\ell}$ is $\mathcal{O}\left(10^{2}\right)$, the correction at the one-loop is still small. In addition, we can tune the parameter $\lambda_{3}$ so that the contributions of the tree-level and one-loop to the $\mathrm{Hhh}$ coupling are canceled to some extent. Meanwhile, the value of $\lambda_{3}$ does not affect the results of the LFU in $\tau$ decay, the muon and electron $g-2$ anomalies.

\section{CONCLUSION}

In the lepton-specific inert two-Higgs-doublet model, we consider relevant theoretical and experimental constraints, especially for the multilepton event searches at the LHC, and discuss the possibilities of explaining the new muon $g-2$ anomaly reported by the Fermilab and electron $g-2$ anomaly. We find that the muon and electron $g-2$ anomalies can be explained simultaneously in the region of $5 \mathrm{GeV}<$ $m_{h}<35 \mathrm{GeV}, 200 \mathrm{GeV}<m_{A}<620 \mathrm{GeV}, 190 \mathrm{GeV}<$ $m_{H^{ \pm}}<620 \mathrm{GeV}, \quad 15<-\kappa_{\mu}<90, \quad 5<\kappa_{\tau}<50, \quad$ and $160<\kappa_{e}<500$.

\section{ACKNOWLEDGMENTS}

This work was supported by the National Natural Science Foundation of China under Grants No. 11975013, No. 11875062, No. 12105248, and by the Project of Shandong Province Higher Educational Science and Technology Program under Grants No. 2019KJJ007.
[1] G. W. Bennett et al. (Muon g-2 Collaboration), Phys. Rev. Lett. 86, 2227 (2001).

[2] G. W. Bennett et al. (Muon g-2 Collaboration), Phys. Rev. D 73, 072003 (2006).

[3] B. Abi et al. (Fermilab Collaboration), Phys. Rev. Lett. 126, 141801 (2021).

[4] T. Aoyama, M. Hayakawa, T. Kinoshita, and M. Nio, Phys. Rev. Lett. 109, 111808 (2012).

[5] A. Czarnecki, W. J. Marciano, and A. Vainshtein, Phys. Rev. D 67, 073006 (2003).

[6] G. Eichmann, C. S. Fischer, and R. Williams, Phys. Rev. D 101, 054015 (2020).

[7] M. Davier, A. Hoecker, B. Malaescu, and Z. Zhang, Eur. Phys. J. C 80, 241 (2020).

[8] R. H. Parker, C. Yu, W. Zhong, B. Estey, and H. Mueller, Science 360, 191 (2018).

[9] D. Hanneke, S. Fogwell, and G. Gabrielse, Phys. Rev. Lett. 100, 120801 (2008).

[10] D. Hanneke, S. F. Hoogerheide, and G. Gabrielse, Phys. Rev. A 83, 052122 (2011).

[11] L. Morel, Z. Yao, P. Clad, and S. Guellati-Khlifa, Nature (London) 588, 61 (2020).

[12] A. Dedes and H. E. Haber, J. High Energy Phys. 05 (2001) 006.

[13] D. Chang, W.-F. Chang, C.-H. Chou, and W.-Y. Keung, Phys. Rev. D 63, 091301 (2001).

[14] K. M. Cheung, C. H. Chou, and O. C. W. Kong, Phys. Rev. D 64, 111301 (2001).

[15] J. Cao, P. Wan, L. Wu, and J. M. Yang, Phys. Rev. D 80, 071701 (2009).

[16] L. Wang and X. F. Han, J. High Energy Phys. 05 (2015) 039.

[17] E. J. Chun, Z. Kang, M. Takeuchi, and Y.-L. Tsai, J. High Energy Phys. 11 (2015) 099.
[18] T. Han, S. K. Kang, and J. Sayre, J. High Energy Phys. 02 (2016) 097.

[19] V. Ilisie, J. High Energy Phys. 04 (2015) 077.

[20] A. Cherchiglia, P. Kneschke, D. Stockinger, and H. StockingerKim, J. High Energy Phys. 01 (2017) 007.

[21] X. Liu, L. Bian, X.-Q. Li, and J. Shu, Nucl. Phys. B909, 507 (2016).

[22] T. Abe, R. Sato, and K. Yagyu, J. High Energy Phys. 07 (2015) 064.

[23] A. Crivellin, J. Heeck, and P. Stoffer, Phys. Rev. Lett. 116, 081801 (2016).

[24] E. J. Chun and J. Kim, J. High Energy Phys. 07 (2016) 110.

[25] L. Wang, J. M. Yang, M. Zhang, and Y. Zhang, Phys. Lett. B 788, 519 (2019).

[26] D. Sabatta, A. S. Cornell, A. Goyal, M. Kumar, and B. Mellado, Chin. Phys. C 44, 063103 (2020).

[27] E. J. Chun and T. Mondal, Phys. Lett. B 802, 135190 (2020).

[28] H. Davoudiasl and W. J. Marciano, Phys. Rev. D 98, 075011 (2018).

[29] J.-J. Zhang, M. He, X.-G. He, and G. Li, Nucl. Phys. B953, 114968 (2020).

[30] A. Crivellin, M. Hoferichter, and P. Schmidt-Wellenburg, Phys. Rev. D 98, 113002 (2018).

[31] J. Liu, C. E. M. Wagner, and X.-P. Wang, J. High Energy Phys. 03 (2019) 008.

[32] X.-F. Han, T. Li, L. Wang, and Y. Zhang, Phys. Rev. D 99, 095034 (2019).

[33] M. Endo and W. Yin, J. High Energy Phys. 08 (2019) 122.

[34] H. Davoudiasl and W. J. Marciano, Phys. Rev. D 98, 075011 (2018).

[35] M. Abdullah, B. Dutta, S. Ghosh, and T. Li, Phys. Rev. D 100, 115006 (2019).

[36] S. Gardner and X. Yan, Phys. Rev. D 102, 075016 (2020). 
[37] M. Badziak and K. Sakurai, J. High Energy Phys. 10 (2019) 024.

[38] A. Hernandez, S. F. King, H. Lee, and S. J. Rowley, Phys. Rev. D 101, 115016 (2020).

[39] A. Hernandez, Y. Velasquez, S. Kovalenko, H. N. Long, N. Perez-Julve, and V. V. Vien, Eur. Phys. J. C 81, 191 (2021).

[40] S. Jana and S. Saad, Phys. Rev. D 101, 115037 (2020).

[41] L. Calibbi, M. Ibanez, A. Melis, and O. Vives, J. High Energy Phys. 06 (2020) 087.

[42] C. Chen and T. Nomura, Nucl. Phys. B964, 115314 (2021).

[43] C. Chua, Phys. Rev. D 102, 055022 (2020).

[44] C. Hati, J. Kriewald, J. Orloff, and A. M. Teixeira, J. High Energy Phys. 07 (2020) 235.

[45] B. Dutta, S. Ghosh, and T. Li, Phys. Rev. D 102, 055017 (2020).

[46] F. J. Botella, F. Cornet-Gomez, and M. Nebot, Phys. Rev. D 102, 035023 (2020).

[47] I. Dorsner, S. Fajfer, and S. Saad, Phys. Rev. D 102, 075007 (2020).

[48] S. Jana, W. Rodejohann, and S. Saad, Phys. Rev. D 102, 075003 (2020).

[49] E. J. Chun and T. Mondal, J. High Energy Phys. 11 (2020) 077.

[50] S. Li, X.-Q. Li, Y. Li, Y.-D. Yang, and X. Zhang, J. High Energy Phys. 01 (2021) 034.

[51] L. D. Rose, S. Khalil, and S. Moretti, Phys. Lett. B 816, 136216 (2021).

[52] A. Hernandez, S. F. King, and H. Lee, Phys. Rev. D 103, 115024 (2021).
[53] N. Chen, B. Wang, and C. Yao, arXiv:2102.05619.

[54] A. Bodas, R. Coy, and S. King, arXiv:2102.07781.

[55] J. Cao, Y. He, J. Lian, D. Zhang, and P. Zhu, Phys. Rev. D 104, 055009 (2021).

[56] Y. Amhis et al. (Heavy Flavor Averaging Group (HFAG) Collaboration), arXiv:1412.7515.

[57] S. Schael et al. (ALEPH, DELPHI, L3, OPAL, SLD, LEP Electroweak Working Group, SLD Electroweak Group, and SLD Heavy Flavour Group Collaborations), Phys. Rep. 427, 257 (2006).

[58] D. Eriksson, J. Rathsman, and O. Stål, Comput. Phys. Commun. 181, 189 (2010).

[59] M. Tanabashi et al. (Particle Data Group), Phys. Rev. D 98 , 030001 (2018).

[60] P. Bechtle, O. Brein, S. Heinemeyer, G. Weiglein, and K. E. Williams, Comput. Phys. Commun. 181, 138 (2010).

[61] J. Alwall et al., J. High Energy Phys. 07 (2014) 079.

[62] P. Torrielli and S. Frixione, J. High Energy Phys. 04 (2010) 110.

[63] J. de Favereau et al. (DELPHES 3 Collaboration), J. High Energy Phys. 02 (2014) 057.

[64] D. Dercks, N. Desai, J. S. Kim, K. Rolbiecki, J. Tattersall, and T. Weber, Comput. Phys. Commun. 221, 383 (2017).

[65] G. Pozzo and Y. Zhang, Phys. Lett. B 789, 582 (2019).

[66] A. M. Sirunyan et al. (CMS Collaboration), Report No. CMS-PAS-SUS-19-012.

[67] J. Abdallah et al. (DELPHI Collaboration), Eur. Phys. J. C 38, 1 (2004). 